\title{
Absence of replica symmetry breaking in disordered FKG-Ising models under uniform field
}

\author{
C. Itoi and Y. Utsunomiya \\ Department of Physics, GS and CST, Nihon University, \\ Kanda-Surugadai, Chiyoda, Tokyo 101-8308, Japan
}

October 16, 2019

\begin{abstract}
We prove that the variance of spin overlap vanishes in disordered Ising models satisfying the Fortuin-Kasteleyn-Ginibre (FKG) inequality under a uniform field, such as generally distributed random field Ising model, site- and bond-diluted Ising models with the Bernoulli distribution. Chatterjee's proof for the Gaussian random field Ising model is generalized to other independent identically distributed quenched disorder under a uniform field.
\end{abstract}

\section{Introduction}

Replica symmetry breaking is a spontaneous symmetry breaking phenomenon in spin models with quenched disorders. This symmetry breaking appears generally in mean field disordered spin models at low temperature, such as Parisi's replica symmetry breaking formula [23, 25] for the Sherrington-Kirkpatrick (SK) model rigorously proven by Talagrand [26]. Krzakala, Ricci-Tersenghi, Sherrington and Zdeborova have pointed out an evidence that an extended spin glass phase does not exist in disordered ferromagnetic spin models satisfying the FortuinKasteleyn-Ginibre (FKG) inequality [11], such as the random field Ising model, diluted Ising model, the random field Ginzburg-Lamdau model and random temperature Ginzburg-Landau model [21, 22]. Recently, Chatterjee has proven that there is no replica symmetry breaking phase in the random field Ising model in an arbitrary dimension rigorously, if the distribution of random field is Gaussian [4]. He has proven that the variance of the spin overlap vanishes for almost all coupling constants in the random field Ising model, which satisfies the FKG inequality [11] and the Ghirlanda-Guerra identities. There have been several studies to generalize Chatterjee's proof and some mathematical results to non-Gaussian distributions of disorders or quantum spin systems. His method has been generalized to quantum systems having the weak FKG property [16]. Auffinger and W.K. Chen have argued disordered spin systems with generalized distribution and have proven that the overlap is self-averaging in the random field Ising model with a weak coupling constant depending on the system size. They also have proven the Ghirlanda-Guerra identities and the ultrametricity in the mixed $p$-spin model with generalized disorder, and has shown disorder and temperature chaos phenomena in both the mixed $p$-spin model and the Edwards-Anderson (EA) model with non-Gaussian distributions. Roldan and Vira have proven the absence of replica symmetry breaking for a different specific class of non-Gaussian distributions in the random field Ising model [24]. Their proof needs the decay of random fields far from the origin of the lattice. Quite recently, Y.T. Chen has improved the arguments by Auffinger and W. K. Chen [1] for the $p$-spin models with a generalized disorder [6]. Barbier, Chan and Macris have proven the concentration of multi-overlaps for random ferromagnetic spin models by a non-trivial application of the Griffiths-Kelly-Sherman correlation inequality [3]. The absence of replica symmetry breaking has been proven also in the 
random field Ginzburg-Landau model with Gaussian random field [19]. In the present paper, we prove that the replica symmetry breaking does not occur in disordered Ising systems under general independent identically distributed (i.i.d.) random fields satisfying the FKG inequality, such as the random field Ising model and bond-diluted Ising model under uniform field. The concentration of the multi-overlap obtained by Barbier, Chan and Macris 3 for the multiplicity $k=2$ is similar to our result but a bit weaker than ours, since we prove it without an assumption of the Griffiths-Kelly-Sherman correlation inequality [13, 14, 20]. To prove it for such models, we employ several new methods as well as that used in Ref[17, 18].

\section{Definitions and main theorem}

\subsection{Hamiltonian of models}

Let $L$ be a positive integer, and define a $d$-dimensional hyper cubic lattice by $\Lambda_{L}:=[1, L]^{d} \cap \mathbb{Z}^{d}$ whose volume is $\left|\Lambda_{L}\right|=L^{d}$. Let $B_{L}:=\left\{\{x, y\} \mid x, y \in \Lambda_{L}, C(x, y)\right\}$ be a collection of interaction bonds, where $C(x, y)$ is a condition on two sites $x, y \in \Lambda_{L}$. Assume that the interaction is shortranged and $\left|B_{L}\right|=C_{1}\left|\Lambda_{L}\right|$ for a certain constant $C_{1} \geq 1$ independent of $L$. For example, $C(x, y)$ is given by $|x-y|=1$ to define the collection of nearest neighbor bonds in $\Lambda_{L}$. In this case, $C_{1}=$ d. Let $C_{L}$ be a collection of interaction ranges defined by $C_{L}:=B_{L}$ or $C_{L}:=\Lambda_{L}$ to construct FKG-Ising systems. Note that $\left|C_{L}\right| \leq C_{1}\left|\Lambda_{L}\right|$. To define Hamiltonian, we introduce random couplings. Let $r:=\left(r_{i}\right)_{i \in C_{L}}$ be real valued i.i.d. random variables with a finite expectation and a finite variance. Let $J:=\left(J_{X}(r)\right)_{X \in B_{L}}$ be a sequence of bond random variables which consist of positive semi-definite valued functions of the i.i.d. random variables $r=\left(r_{i}\right)_{i \in C_{L}}$, such that $J_{X}(r)$ and $J_{Y}(r)$ are independent if $X \cap Y=\phi$ for two sets $X, Y \in C_{L}$. Let $\left(h_{x}(r)\right)_{x \in \Lambda_{L}}$ be a sequence of site random variables which consist of functions of i.i.d. random variables $r=\left(r_{i}\right)_{i \in C_{L}}$, such that $h_{x}(r)$ and $h_{y}(r)$ are independent if $x \neq y$. Define Hamiltonian as a function of the sequence $r$ of random variables and spin configurations $\sigma \in \Sigma_{L}:=\{-1,1\}^{\Lambda_{L}}$ on the lattice $\Lambda_{L}$.

$$
H(r, \sigma)=-\sum_{X \in B_{L}} J_{X}(r) \sigma_{X}-\sum_{x \in \Lambda_{L}} h_{x}(r) \sigma_{x}
$$

\section{$2.2 \quad$ Examples}

Here we introduce several examples in our definition of the Hamiltonians in general models.

1. Random field Ising model This model is defined by deterministic bond couplings $J_{X}(r)=1$ for any $X \in B_{L}$ and site couplings $h_{x}(r)=b r_{x}+h$ for any $x \in C_{L}:=\Lambda_{L}$ for $(b, h) \in \mathbb{R}^{2}$, where $\left|\Lambda_{L}\right|=L^{d}$.

$$
H_{\mathrm{RFI}}(r, \sigma)=-\sum_{X \in B_{L}} \sigma_{X}-\sum_{x \in \Lambda_{L}}\left(b r_{x}+h\right) \sigma_{x}
$$

where each random variable $r_{x}$ at $x \in \Lambda_{L}$ satisfies a certain distribution with zero expectation and a finite variance.

2. Bond-diluted Ising model This model is defined by random bond coupling $J_{X}(r)=J r_{X}$ for $J>0$ and deterministic site coupling $h_{x}(r)=h \in \mathbb{R}$ for $X \in C_{L}:=B_{L}$

$$
H_{\mathrm{BDI}}(r, \sigma)=-J \sum_{X \in B_{L}} r_{X} \sigma_{X}-\sum_{x \in \Lambda_{L}} h \sigma_{x},
$$

where the random variable $r_{X}$ for $X \in B_{L}$ satisfies the Bernoulli distribution for $0<p<1$

$$
p\left(r_{X}\right)=p \delta\left(r_{X}-1\right)+(1-p) \delta\left(r_{X}\right) .
$$


Note that $J_{X}(r)$ and $J_{Y}(r)$ are independent if and only if $X \neq Y$. The variance of each $r_{X}$ is $v=p(1-p)$ in this distribution.

3. Site-diluted Ising model Spins are missing at several sites in the site-diluted Ising model. This model has random bond coupling $J_{X}(r)=J \prod_{x \in X} r_{x}$ for $X \in B_{L}$ and random site coupling $h_{x}=h r_{x}$ for $x \in C_{L}:=\Lambda_{L}$ with $h \in \mathbb{R}$, where $\left|\Lambda_{L}\right|=L^{d}$. In this model, $J_{X}(r)$ and $J_{Y}(r)$ are independent if and only if $X \cap Y=\phi$. The Hamiltonian of the site-diluted Ising model is defined by

$$
H_{\mathrm{SDI}}(r, \sigma)=-J \sum_{X \in B_{L}} r_{X} \sigma_{X}-\sum_{x \in \Lambda_{L}} h r_{x} \sigma_{x}
$$

where the the random variable $r_{x}$ at $x \in \Lambda_{L}$ satisfies the Bernoulli distribution for $0<p<1$

$$
p\left(r_{x}\right)=p \delta\left(r_{x}-1\right)+(1-p) \delta\left(r_{x}\right) .
$$

\subsection{The Gibbs state}

Here, we define Gibbs state for the Hamiltonian. The partition function as a function of $(\beta, h) \in$ $[0, \infty) \times \mathbb{R}$ and a sequence $r=\left(r_{i}\right)_{i \in C_{L}}$ is defined by

$$
Z_{L}(\beta, h, r):=\sum_{\sigma \in \Sigma_{L}} e^{-\beta H(r, \sigma)}
$$

The expectation of a function of spin configuration $f(\sigma)$ in the Gibbs state is given by

$$
\langle f(\sigma)\rangle=\frac{1}{Z_{L}(\beta, h, r)} \sum_{\sigma \in \Sigma_{L}} f(\sigma) e^{-\beta H(r, \sigma)} .
$$

Define a function of $\beta \in[0, \infty)$ and a sequence $J=\left(r_{i}\right)_{i \in C_{L}}$ by

$$
\psi_{L}(\beta, r):=\frac{1}{\left|\Lambda_{L}\right|} \log Z_{L}(\beta, h, r)
$$

$-\frac{\left|\Lambda_{L}\right|}{\beta} \psi_{L}(\beta, r)$ is called free energy in statistical physics. The following function $p_{L}:[0, \infty) \rightarrow \mathbb{R}$ is defined by the expectation of $\psi_{L}(\beta, h, r)$ over $r$

$$
p_{L}(\beta, h, v):=\mathbb{E} \psi_{L}(\beta, h, r),
$$

where $\mathbb{E}$ denotes the expectation over the i.i.d. random variables $r$ with a variance $v$.

\subsection{Replica symmetry}

Next, we explain replica symmetry breaking phenomena which apparently violate self-averaging of the overlap between two replicated quantities in a replica symmetric expectation. Let $\sigma^{a}(a=$ $1, \cdots, n)$ be $n$ replicated spin configurations, and consider the following Hamiltonian

$$
H\left(r, \sigma^{1}, \cdots, \sigma^{n}\right):=\sum_{a=1}^{n} H\left(r, \sigma^{a}\right),
$$

where replicated spin configurations share the same quenched randomness $r$. This Hamiltonian is invariant under an arbitrary permutation $s\left(\in S_{n}\right)$, which is a bijection acting on $\{1,2, \cdots, n\}$.

$$
H\left(r, \sigma^{1}, \cdots, \sigma^{n}\right)=H\left(r, \sigma^{s 1}, \cdots, \sigma^{s n}\right)
$$


This permutation symmetry is the replica symmetry. The spin overlap $R_{a, b}$ between two replicated spin configurations is defined by

$$
R_{a, b}:=\frac{1}{\left|\Lambda_{L}\right|} \sum_{x \in \Lambda_{L}} \sigma_{x}^{a} \sigma_{x}^{b} .
$$

for all models except for the site-diluted Ising model. In this exceptional model, the spin overlap is defined by

$$
R_{a, b}:=\frac{1}{\left|\Lambda_{L}\right|} \sum_{x \in \Lambda_{L}} r_{x}^{2} \sigma_{x}^{a} \sigma_{x}^{b} .
$$

If the distribution of the overlap has a finite variance, it implies the replica symmetry breaking as observed in the Sherrington-Kirkpatrick model [23, 26, 27]. In Chatterjee's definition [4], we say that the replica symmetry breaking occurs if the finite variance calculated in the replica symmetric expectation in the infinite-volume limit

$$
\lim _{L \rightarrow \infty} \mathbb{E}\left\langle\Delta R_{1,2}^{2}\right\rangle \neq 0
$$

where $\Delta R_{1,2}:=R_{1,2}-\mathbb{E}\left\langle R_{1,2}\right\rangle$. Chatterjee has given this definition of the replica symmetry breaking and proven

$$
\lim _{L \rightarrow \infty} \mathbb{E}\left\langle\Delta R_{1,2}^{2}\right\rangle=0,
$$

in the Gaussian random field Ising model [4]. In the present paper, we extend his proof to non-Gaussian disordered Ising systems with the FKG property under a uniform field.

\subsection{Main theorem}

Consider a model satisfying the following assumptions.

Assumption 1 The infinite-volume limit

$$
\lim _{L \rightarrow \infty} p_{L}(\beta, h)=p(\beta, h)
$$

exists for each $\beta \in[0, \infty) \times \mathbb{R}$.

Assumption 2 Let $f, g$ be monotonically increasing function of spin configuration $\sigma \in \Sigma_{L}$. The FKG inequality [11]

$$
\langle f(\sigma) ; g(\sigma)\rangle:=\langle f(\sigma) g(\sigma)\rangle-\langle f(\sigma)\rangle\langle g(\sigma)\rangle \geq 0
$$

is valid for any $(\beta, h) \in[0, \infty) \times \mathbb{R}$ and for an arbitrarily fixed random sequence $r$.

Assumption 3 The expectation and the variance of random variables $J_{X}(r)$ for $X \in B_{L}$ and $h_{x}(r)$ for $x \in \Lambda_{L}$ exist as a finite value.

Note that Assumption 1 is proven for a class of models with random short-range interactions [4, 10]. Under these Assumptions, we prove the following theorem.

Theorem 2.1 In a model defined by the Hamiltonian (1) satisfying Assumptions 1, 2 and 3 the following variance vanishes

$$
\lim _{L \rightarrow \infty}\left[\mathbb{E}\left\langle R_{1,2}{ }^{2}\right\rangle-\left(\mathbb{E}\left\langle R_{1,2}\right\rangle\right)^{2}\right]=0,
$$

for almost all $(\beta, h) \in[0, \infty) \times \mathbb{R}$ in the infinite-volume limit. 


\section{Proof}

\subsection{Variance inequalities}

To prove Theorem 2.1, we introduce an artificial Gaussian random field perturbing the original Hamiltonian.

Definition 3.1 Let $g=\left(g_{x}\right)_{x \in \Lambda_{L}}$ be a sequence of i.i.d. standard Gaussian random variables and define a function of $g$ and $\sigma \in \Sigma_{L}$ by

$$
\xi_{L}(g, \sigma):=\frac{1}{\left|\Lambda_{L}\right|} \sum_{x \in \Lambda_{L}} g_{x} \sigma_{x}
$$

For the exceptional case in the site-diluted Ising model,

$$
\xi_{L}(g, \sigma):=\frac{1}{\left|\Lambda_{L}\right|} \sum_{x \in \Lambda_{L}} g_{x} r_{x} \sigma_{x}
$$

Define the following perturbed Hamiltonian

$$
H_{\mu}(q, \sigma):=H(r, \sigma)-\mu\left|\Lambda_{L}\right| \xi_{L}(g, \sigma)
$$

with a coupling constant $\mu \in \mathbb{R}$. Let $q=\left(q_{i}\right)_{i=1,2, \cdots, N}:=\left(g_{x}, r_{i}\right)_{x \in \Lambda_{L}, i \in C_{L}}$ be a combined sequence of random variables labeled by numbers $i=1, \cdots, N$, and $\mathbb{E}$ denotes the expectation over all random variables $\left(q_{i}\right)_{i=1, \cdots, N}$. Define a partition function of the perturbed Hamiltonian

$$
Z_{L}(\beta, h, \mu, q):=\sum_{\sigma \in \Sigma_{L}} e^{-\beta H_{\mu}}
$$

Define the corresponding functions

$$
\begin{aligned}
& \psi_{L}(\beta, h, \mu, q):=\frac{1}{\left|\Lambda_{L}\right|} \log Z_{L}(\beta, h, \mu, q), \\
& p_{L}(\beta, h, \mu):=\mathbb{E} \psi_{L}(\beta, h, \mu, q), \\
& p(\beta, h, \mu):=\lim _{L \rightarrow \infty} p_{L}(\beta, h, \mu) .
\end{aligned}
$$

And denote the expectation of an arbitrary function $f(\sigma)$ of spin configuration $\sigma \in \Sigma_{L}$ in the perturbed Gibbs state with this Hamiltonian by

$$
\langle f(\sigma)\rangle_{\mu}:=\frac{1}{Z_{L}(\beta, h, \mu, q)} \sum_{\sigma \in \Sigma_{L}} f(\sigma) e^{-\beta H_{\mu}(q, \sigma)}
$$

Note that this model defined by the above Hamiltonian satisfies the FKG property still.

Lemma 3.2 There exists a positive number $C$ independent of the system size $L$, such that the variance of $\psi_{L}$ is bounded by

$$
\mathbb{E} \psi_{L}(\beta, h, \mu, q)^{2}-p_{L}(\beta, h, \mu)^{2} \leq \frac{C}{\left|\Lambda_{L}\right|},
$$

for any $(\beta, h, \mu) \in(0, \infty) \times \mathbb{R}^{2}$.

Proof. For an integer $m=1,2, \cdots, N$ define a symbol $\mathbb{E}_{m}$ which denotes the expectation over random variables $\left(q_{j}\right)_{j>m}$. Note that $\mathbb{E}_{0}=\mathbb{E}$ is the expectation over the all random variables $q=\left(q_{j}\right)_{j=1,2, \cdots, N}$, and $\mathbb{E}_{N}$ is the identity mapping. 
Here, we represent $\psi_{L}(h)$ as a function of a sequence of random variables $q=\left(q_{j}\right)_{j=1, \cdots, N}$ for lighter notation.

$$
\begin{aligned}
& \mathbb{E} \psi_{L}(q)^{2}-\left(\mathbb{E} \psi_{L}(q)\right)^{2} \\
& =\mathbb{E}\left(\mathbb{E}_{N} \psi_{L}(q)\right)^{2}-\mathbb{E}\left(\mathbb{E}_{0} \psi_{L}(q)\right)^{2} \\
& =\sum_{m=1}^{N} \mathbb{E}\left[\left(\mathbb{E}_{m} \psi_{L}(q)\right)^{2}-\left(\mathbb{E}_{m-1} \psi_{L}(q)\right)^{2}\right]
\end{aligned}
$$

In the $m$-th term, regard $\psi_{L}\left(q_{m}\right)$ as a function of $q_{m}$. Let $q_{m}^{\prime}$ be an independent random variable satisfying the same distribution as that of $q_{m}$, and $\mathbb{E}^{\prime}$ denotes an expectation over only $q_{m}^{\prime}$. Note that $\mathbb{E}_{m-1} \psi_{L}\left(q_{1}, \cdots, q_{m}, \cdots, q_{N}\right)=\mathbb{E}_{m} \mathbb{E}^{\prime} \psi_{L}\left(q_{1}, \cdots, q_{m}^{\prime}, \cdots, q_{N}\right)$. Let $C_{m} \subset C_{L}$ be a collection of all $X \in C_{L}$ such that $J_{X}(q)$ depends on $q_{m}$.

$$
\begin{aligned}
& \mathbb{E}\left[\left(\mathbb{E}_{m} \psi_{L}\left(q_{m}\right)\right)^{2}-\left(\mathbb{E}_{m-1} \psi_{L}\left(q_{m}\right)\right)^{2}\right] \\
& =\mathbb{E}\left[\left(\mathbb{E}_{m} \psi_{L}\left(q_{m}\right)\right)^{2}-\left(\mathbb{E}_{m} \mathbb{E}^{\prime} \psi_{L}\left(q_{m}^{\prime}\right)\right)^{2}\right] \\
& =\mathbb{E}\left[\mathbb{E}_{m}\left(\psi_{L}\left(q_{m}\right)-\mathbb{E}^{\prime} \psi_{L}\left(q_{m}^{\prime}\right)\right)\right]^{2} \\
& =\mathbb{E}\left[\mathbb{E}_{m} \mathbb{E}^{\prime}\left(\psi_{L}\left(q_{m}\right)-\psi_{L}\left(q_{m}^{\prime}\right)\right)\right]^{2} \\
& =\mathbb{E}\left[\mathbb{E}_{m} \mathbb{E}^{\prime} \psi_{L}\left(q_{m}\right)-\psi_{L}\left(q_{m}^{\prime}\right)\right]^{2}, \\
& =\mathbb{E}\left[\mathbb{E}_{m} \mathbb{E}^{\prime} \int_{q_{m}^{\prime}}^{q_{m}} d s \frac{\partial}{\partial s} \psi_{L}(s)\right]^{2}, \\
& =\frac{1}{\left|\Lambda_{L}\right|^{2}} \mathbb{E}\left[\mathbb{E}_{m} \mathbb{E}^{\prime} \int_{q_{m}^{\prime}}^{q_{m}} d s \beta \sum_{X \in C_{m}} \frac{\partial J_{X}(s)}{\partial s}\left\langle\sigma_{X}\right\rangle_{s}\right]^{2} \\
& =\frac{1}{\left|\Lambda_{L}\right|^{2}} \mathbb{E}\left[\mathbb{E}_{m} \mathbb{E}^{\prime} \beta \sum_{X \in C_{m}} \int_{J_{X}\left(q_{m}^{\prime}\right)}^{J_{X}\left(q_{m}\right)} d J_{X}\left\langle\sigma_{X}\right\rangle_{s}\right]^{2} \\
& \leq \frac{1}{\left|\Lambda_{L}\right|^{2}} \mathbb{E}\left[\mathbb{E}^{\prime} \beta \sum_{X \in C_{m}}\left|J_{X}\left(q_{m}^{\prime}\right)-J_{X}\left(q_{m}\right)\right|\right]^{2} \leq \frac{C^{\prime}}{N^{2}}
\end{aligned}
$$

where $C^{\prime}$ is a positive number independent of $N$, and we denote the Gibbs expectation in the conditional probability under $q_{m}$ by

$$
\langle f(\sigma)\rangle_{q_{m}}=\frac{1}{Z_{L}\left(q_{m}\right)} \sum_{\sigma \in \Sigma_{L}} f(\sigma) e^{-\beta H\left(q_{m}, \sigma\right)} .
$$

Since $\left|\Lambda_{L}\right| \leq\left|C_{L}\right| \leq C_{1}\left|\Lambda_{L}\right|$, we have $2\left|\Lambda_{L}\right| \leq N \leq\left(C_{1}+1\right)\left|\Lambda_{L}\right|$ and therefore

$$
\mathbb{E} \psi_{L}(q)^{2}-\left(\mathbb{E} \psi_{L}(q)\right)^{2} \leq \frac{C^{\prime}}{2\left|\Lambda_{L}\right|}
$$

This completes the proof.

To show the existence of the infinite-volume limit of the expectation of the overlap, we use the standard convexity argument [27].

Lemma 3.3 For almost all $\mu \in \mathbb{R} \backslash\{0\}$, the following expectation of the overlap exists in the infinite-volume limit, and it is represented in terms of the derivative of $p(\beta, h, \mu)$

$$
\lim _{L \rightarrow \infty} \mathbb{E}\left\langle R_{1,2}\right\rangle_{\mu}=1-\frac{1}{\beta^{2} \mu} \frac{\partial p}{\partial \mu} .
$$

Proof. Here we regard $p_{L}(\mu)$ and $p(\mu)$ as functions of $\mu$. Define a function $e_{L}(\epsilon, \mu)$ of $\epsilon>0$ and $\mu \in \mathbb{R}$

$$
e_{L}(\epsilon, \mu):=\frac{1}{\epsilon}\left[\left|p_{L}(\mu+\epsilon)-p(\mu+\epsilon)\right|+\left|p_{L}(\mu-\epsilon)-p(\mu-\epsilon)\right|+\left|p_{L}(\mu)-p(\mu)\right|\right] .
$$


Since $p_{L}$ and $p$ are convex functions of $\mu$, we have

$$
\begin{aligned}
& \frac{\partial p_{L}}{\partial \mu}(\mu)-\frac{\partial p}{\partial \mu}(\mu) \leq \frac{1}{\epsilon}\left[p_{L}(\mu+\epsilon)-p_{L}(\mu)\right]-\frac{\partial p}{\partial \mu} \\
& \leq \frac{1}{\epsilon}\left[p_{L}(\mu+\epsilon)-p_{L}(\mu)-p(\mu+\epsilon)+p(\mu+\epsilon)+p(\mu)-p(\mu)\right]-\frac{\partial p}{\partial \mu}(\mu) \\
& \leq \frac{1}{\epsilon}\left[\left|p_{L}(\mu+\epsilon)-p(\mu+\epsilon)\right|+\left|p(\mu)-p_{L}(\mu)\right|+\frac{1}{\epsilon}[p(\mu+\epsilon)-p(\mu)]-\frac{\partial p}{\partial \mu}(\mu)\right. \\
& \leq e_{L}(\epsilon, \mu)+\frac{\partial p}{\partial \mu}(\mu+\epsilon)-\frac{\partial p}{\partial \mu}(\mu)
\end{aligned}
$$

As in the same calculation, we have

$$
\begin{aligned}
& \frac{\partial p_{L}}{\partial \mu}(\mu)-\frac{\partial p}{\partial \mu}(\mu) \geq \frac{1}{\epsilon}\left[p_{L}(\mu)-p_{L}(\mu-\epsilon)\right]-\frac{\partial p}{\partial \mu}(\mu) \\
& \geq-e_{L}(\epsilon, \mu)+\frac{\partial p}{\partial \mu}(\mu-\epsilon)-\frac{\partial p}{\partial \mu}(\mu)
\end{aligned}
$$

Both inequalities imply

$$
\left|\frac{\partial p_{L}}{\partial \mu}(\mu)-\frac{\partial p}{\partial \mu}(\mu)\right| \leq e_{L}(\epsilon, \mu)+\frac{\partial p}{\partial \mu}(\mu+\epsilon)-\frac{\partial p}{\partial \mu}(\mu-\epsilon) .
$$

In the infinite-volume limit, $e_{L}(\epsilon, \mu)$ vanishes for an arbitrary $\epsilon>0$ by Assumption 1,

$$
\begin{aligned}
& \lim _{L \rightarrow \infty}\left|\beta^{2} \mu\left(1-\frac{1}{\left|\Lambda_{L}\right|} \sum_{x \in \Lambda_{L}} \mathbb{E}\left\langle\sigma_{x}\right\rangle_{\mu}^{2}\right)-\frac{\partial p}{\partial \mu}(\mu)\right| \\
& =\lim _{L \rightarrow \infty}\left|\beta \mathbb{E}\left\langle\xi_{L}\right\rangle_{\mu}-\frac{\partial p}{\partial \mu}(\mu)\right|=\lim _{L \rightarrow \infty}\left|\frac{\partial p_{L}}{\partial \mu}(\mu)-\frac{\partial p}{\partial \mu}(\mu)\right| \leq \frac{\partial p}{\partial \mu}(\mu+\epsilon)-\frac{\partial p}{\partial \mu}(\mu-\epsilon) .
\end{aligned}
$$

Since the convex function $p(\mu)$ is continuously differentiable almost everywhere and $\epsilon>0$ is arbitrary, this limit vanishes for almost all $\mu \in \mathbb{R} \backslash\{0\}$. This completes the proof.

To show the following bound on the Hamiltonian density, we use the standard convexity argument also [27].

Lemma 3.4 For almost all $\mu \in \mathbb{R}$, the following infinite-volume limit vanishes

$$
\lim _{L \rightarrow \infty} \mathbb{E}\left|\left\langle\xi_{L}\right\rangle_{\mu}-\mathbb{E}\left\langle\xi_{L}\right\rangle_{\mu}\right|=0
$$

Proof. Here we regard $\psi_{L}(\mu), p_{L}(\mu)$ and $p(\mu)$ as functions of $\mu$. Define a function $w_{L}(\epsilon, \mu)$ of $\epsilon>0$ and $\mu$.

$$
\begin{aligned}
w_{L}(\epsilon, \mu) & :=\frac{\left|\psi_{L}(\mu+\epsilon)-p_{L}(\mu+\epsilon)\right|}{\epsilon}+\frac{\left|\psi_{L}(\mu)-p_{L}(\mu)\right|}{\epsilon} \\
& +\frac{\left|\psi_{L}(\mu-\epsilon)-p_{L}(\mu-\epsilon)\right|}{\epsilon} .
\end{aligned}
$$

Lemma $\underline{3.2}$ implies

$$
\begin{aligned}
& \mathbb{E} w_{L}(\epsilon, \mu) \\
& \leq \frac{1}{\epsilon} \sqrt{\mathbb{E}\left[\psi_{L}(\mu+\epsilon)-p_{L}(\mu+\epsilon)\right]^{2}}+\frac{1}{\epsilon} \sqrt{\mathbb{E}\left[\psi_{L}(\mu)-p_{L}(\mu)\right]^{2}} \\
& +\frac{1}{\epsilon} \sqrt{\mathbb{E}\left[\psi_{L}(\mu-\epsilon)-p_{L}(\mu-\epsilon)\right]^{2}} \\
& \leq \frac{3}{\epsilon} \sqrt{\frac{C}{\left|\Lambda_{L}\right|}}
\end{aligned}
$$


Since $\psi_{L}$ and $p_{L}$ are convex functions of $\mu$, we have

$$
\begin{aligned}
& \frac{\partial \psi_{L}}{\partial \mu}(\mu)-\frac{\partial p_{L}}{\partial \mu}(\mu) \leq \frac{1}{\epsilon}\left[\psi_{L}(\mu+\epsilon)-\psi_{L}(\mu)\right]-\frac{\partial p_{L}}{\partial \mu} \\
& \leq \frac{1}{\epsilon}\left[\psi_{L}(\mu+\epsilon)-p_{L}(\mu+\epsilon)+p_{L}(\mu+\epsilon)-p_{L}(\mu)+p_{L}(\mu)-\psi_{L}(\mu)\right]-\frac{\partial p}{\partial \mu}(\mu) \\
& \leq \frac{1}{\epsilon}\left[\left|\psi_{L}(\mu+\epsilon)-p_{L}(\mu+\epsilon)\right|+\left|p_{L}(\mu)-\psi_{L}(\mu)\right|+\frac{1}{\epsilon}\left[p_{L}(\mu+\epsilon)-p_{L}(\mu)\right]-\frac{\partial p_{L}}{\partial \mu}(\mu)\right. \\
& \leq w_{L}(\epsilon, \mu)+\frac{\partial p_{L}}{\partial \mu}(\mu+\epsilon)-\frac{\partial p_{L}}{\partial \mu}(\mu) .
\end{aligned}
$$

As in the same calculation, we have

$$
\begin{aligned}
& \frac{\partial \psi_{L}}{\partial \mu}(\mu)-\frac{\partial p_{L}}{\partial \mu}(\mu) \geq \frac{1}{\epsilon}\left[\psi_{L}(\mu)-\psi_{L}(\mu-\epsilon)\right]-\frac{\partial p_{L}}{\partial \mu}(\mu) \\
& \geq-w_{L}(\epsilon, \mu)+\frac{\partial p_{L}}{\partial \mu}(\mu-\epsilon)-\frac{\partial p_{L}}{\partial \mu}(\mu) .
\end{aligned}
$$

Both inequalities imply

$$
\left|\frac{\partial \psi_{L}}{\partial \mu}(\mu)-\frac{\partial p_{L}}{\partial \mu}(\mu)\right| \leq w_{L}(\epsilon, \mu)+\frac{\partial p_{L}}{\partial \mu}(\mu+\epsilon)-\frac{\partial p_{L}}{\partial \mu}(\mu-\epsilon) .
$$

Then, we have

$$
\begin{aligned}
& \mathbb{E}\left|\frac{\partial \psi_{L}}{\partial \mu}(\mu)-\frac{\partial p_{L}}{\partial \mu}(\mu)\right| \leq \mathbb{E} w_{L}(\epsilon, \mu)+\frac{\partial p_{L}}{\partial \mu}(\mu+\epsilon)-\frac{\partial p_{L}}{\partial \mu}(\mu-\epsilon) \\
& \leq \frac{3}{\epsilon} \sqrt{\frac{C}{\left|\Lambda_{L}\right|}}+\frac{\partial p_{L}}{\partial \mu}(\mu+\epsilon)-\frac{\partial p_{L}}{\partial \mu}(\mu-\epsilon) .
\end{aligned}
$$

This and the bound (49) imply

$$
\begin{aligned}
& \beta \int_{\mu_{1}}^{\mu_{2}} d \mu \mathbb{E}\left|\left\langle\xi_{L}\right\rangle_{\mu}-\mathbb{E}\left\langle\xi_{L}\right\rangle_{\mu}\right| \\
& \leq \frac{3\left(\mu_{2}-\mu_{1}\right)}{\epsilon} \sqrt{\frac{C}{\left|\Lambda_{L}\right|}}+\int_{\mu_{2}-\epsilon}^{\mu_{2}+\epsilon} d \mu p_{L}^{\prime}(\mu)-\int_{\mu_{1}-\epsilon}^{\mu_{1}+\epsilon} d \mu p_{L}^{\prime}(\mu) \\
& =\frac{3\left(\mu_{2}-\mu_{1}\right)}{\epsilon} \sqrt{\frac{C}{\left|\Lambda_{L}\right|}}+\int_{\mu_{2}-\epsilon}^{\mu_{2}+\epsilon} d \mu \frac{1}{\left|\Lambda_{L}\right|} \sum_{x \in \Lambda_{L}} \mathbb{E} g_{x}\left\langle\sigma_{x}\right\rangle_{\mu} \\
& -\int_{\mu_{1}-\epsilon}^{\mu_{1}+\epsilon} d \mu \frac{1}{\left|\Lambda_{L}\right|} \sum_{x \in \Lambda_{L}} \mathbb{E} g_{x}\left\langle\sigma_{x}\right\rangle_{\mu} \\
& =\frac{3\left(\mu_{2}-\mu_{1}\right)}{\epsilon} \sqrt{\frac{C}{\left|\Lambda_{L}\right|}}+\int_{\mu_{2}-\epsilon}^{\mu_{2}+\epsilon} d \mu \frac{\beta \mu}{\left|\Lambda_{L}\right|} \sum_{x \in \Lambda_{L}} \mathbb{E}\left(1-\left\langle\sigma_{x}\right\rangle_{\mu}^{2}\right) \\
& -\int_{\mu_{1}-\epsilon}^{\mu_{1}+\epsilon} d \mu \frac{\beta \mu}{\left|\Lambda_{L}\right|} \sum_{x \in \Lambda_{L}} \mathbb{E}\left(1-\left\langle\sigma_{x}\right\rangle_{\mu}^{2}\right) \\
& \leq \frac{3\left(\mu_{2}-\mu_{1}\right)}{\epsilon} \sqrt{\frac{C}{\left|\Lambda_{L}\right|}}+2 \beta\left(\left|\mu_{2}\right|+\left|\mu_{1}\right|\right) \epsilon .
\end{aligned}
$$

Since the above bound is valid for an arbitrary $\epsilon>0$, the right-hand side is bounded by $\left|\Lambda_{L}\right|^{-\frac{1}{4}}$ times a positive number for $\epsilon=\left|\Lambda_{L}\right|^{-\frac{1}{4}}$. Thus, the following infinite-volume limit vanishes

$$
\lim _{L \rightarrow \infty} \int_{\mu_{1}}^{\mu_{2}} d \mu \mathbb{E}\left|\left\langle\xi_{L}\right\rangle_{\mu}-\mathbb{E}\left\langle\xi_{L}\right\rangle_{\mu}\right|=0 .
$$


The integrand vanishes for almost all $\mu \in \mathbb{R}$, since the integration interval $\left(\mu_{1}, \mu_{2}\right)$ is arbitrary and the integrand has a uniform bound

$$
\begin{aligned}
& \mathbb{E}\left|\left\langle\xi_{L}\right\rangle_{\mu}-\mathbb{E}\left\langle\xi_{L}\right\rangle_{\mu}\right| \leq \sqrt{\mathbb{E}\left(\left\langle\xi_{L}\right\rangle_{\mu}-\mathbb{E}\left\langle\xi_{L}\right\rangle_{\mu}\right)^{2}}=\sqrt{\mathbb{E}\left\langle\xi_{L}\right\rangle_{\mu}^{2}-\left(\mathbb{E}\left\langle\xi_{L}\right\rangle_{\mu}\right)^{2}} \\
& =\sqrt{\frac{1}{\left|\Lambda_{L}\right|^{2}} \sum_{x, y \in \Lambda_{L}}\left[\mathbb{E} g_{x} g_{y}\left\langle\sigma_{x}\right\rangle_{\mu}\left\langle\sigma_{y}\right\rangle_{\mu}-\mathbb{E} g_{x}\left\langle\sigma_{x}\right\rangle_{\mu} \mathbb{E} g_{y}\left\langle\sigma_{y}\right\rangle_{\mu}\right]} \\
& =\sqrt{\frac{1}{\left|\Lambda_{L}\right|^{2}} \sum_{x, y \in \Lambda_{L}}\left[\mathbb{E}\left(\frac{\partial^{2}}{\partial g_{x} \partial g_{y}}+\delta_{x, y}\right)\left\langle\sigma_{x}\right\rangle_{\mu}\left\langle\sigma_{y}\right\rangle_{\mu}-\mathbb{E} \frac{\partial\left\langle\sigma_{x}\right\rangle_{\mu}}{\partial g_{x}} \mathbb{E} \frac{\partial\left\langle\sigma_{y}\right\rangle_{\mu}}{\partial g_{y}}\right]} \\
& =\sqrt{\frac{1}{\left|\Lambda_{L}\right|^{2}} \sum_{x, y \in \Lambda_{L}} \mathbb{E}\left[\beta^{2} \mu^{2}\left\langle\sigma_{x} ; \sigma_{y}\right\rangle_{\mu}\left(\left\langle\sigma_{x} \sigma_{y}\right\rangle_{\mu}-5\left\langle\sigma_{x}\right\rangle_{\mu}\left\langle\sigma_{y}\right\rangle_{\mu}\right)+\delta_{x, y}\left\langle\sigma_{x}\right\rangle_{\mu}^{2}\right]} \\
& \leq \sqrt{12 \beta^{2} \mu^{2}+\left|\Lambda_{L}\right|^{-1}} \leq \sqrt{12 \beta^{2} \mu^{2}+1} .
\end{aligned}
$$

Also in the exceptional case defined by (18), this is proven in the same way. This completes the proof.

Lemma 3.5 The variance of the overlap in the Gibbs state vanishes for any $\mu \in \mathbb{R}$ for almost all $h \in \mathbb{R}$ for an arbitrary sequence of random variables $q=\left(q_{i}\right)_{i=1, \cdots, N}$ for any $\beta \in[0, \infty)$

$$
\lim _{L \rightarrow \infty}\left[\left\langle R_{1,2}^{2}\right\rangle_{\mu}-\left\langle R_{1,2}\right\rangle_{\mu}^{2}\right]=0 .
$$

Proof. The following variance of the overlap defined by (11) is evaluated in terms of correlation function

$$
\begin{aligned}
& \left\langle R_{1,2}^{2}\right\rangle_{\mu}-\left\langle R_{1,2}\right\rangle_{\mu}^{2}=\frac{1}{\left|\Lambda_{L}\right|^{2}} \sum_{x, y \in \Lambda}\left(\left\langle\sigma_{x} \sigma_{y}\right\rangle_{\mu}^{2}-\left\langle\sigma_{x}\right\rangle_{\mu}^{2}\left\langle\sigma_{y}\right\rangle_{\mu}^{2}\right) \\
& =\frac{1}{\left|\Lambda_{L}\right|^{2}} \sum_{x, y \in \Lambda}\left(\left\langle\sigma_{x} \sigma_{y}\right\rangle_{\mu}-\left\langle\sigma_{x}\right\rangle_{\mu}\left\langle\sigma_{y}\right\rangle_{\mu}\right)\left(\left\langle\sigma_{x} \sigma_{y}\right\rangle_{\mu}+\left\langle\sigma_{x}\right\rangle_{\mu}\left\langle\sigma_{y}\right\rangle_{\mu}\right) \\
& \leq \frac{1}{\left|\Lambda_{L}\right|^{2}} \sum_{x, y \in \Lambda}\left|\left\langle\sigma_{x} \sigma_{y}\right\rangle_{\mu}-\left\langle\sigma_{x}\right\rangle_{\mu}\left\langle\sigma_{y}\right\rangle_{\mu}\right|\left|\left\langle\sigma_{x} \sigma_{y}\right\rangle_{\mu}+\left\langle\sigma_{x}\right\rangle_{\mu}\left\langle\sigma_{y}\right\rangle_{\mu}\right| \\
& \leq \frac{2}{\left|\Lambda_{L}\right|^{2}} \sum_{x, y \in \Lambda}\left|\left\langle\sigma_{x} \sigma_{y}\right\rangle_{\mu}-\left\langle\sigma_{x}\right\rangle_{\mu}\left\langle\sigma_{y}\right\rangle_{\mu}\right| \\
& =\frac{2}{\left|\Lambda_{L}\right|^{2}} \sum_{x, y \in \Lambda}\left(\left\langle\sigma_{x} \sigma_{y}\right\rangle_{\mu}-\left\langle\sigma_{x}\right\rangle_{\mu}\left\langle\sigma_{y}\right\rangle_{\mu}\right) \\
& =\frac{2}{\left|\Lambda_{L}\right|^{2} \beta} \sum_{x \in \Lambda} \frac{\partial}{\partial h}\left\langle\sigma_{x}\right\rangle_{\mu} .
\end{aligned}
$$

The FKG inequality has been used. Therefore, the integralation over $h \in\left(h_{1}, h_{2}\right)$ in an arbitrary interval becomes

$$
\int_{h_{1}}^{h_{2}} d h\left(\left\langle R_{1,2}^{2}\right\rangle_{\mu, h}-\left\langle R_{1,2}\right\rangle_{\mu, h}^{2}\right) \leq \frac{2}{\beta\left|\Lambda_{L}\right|^{2}} \sum_{x \in \Lambda}\left[\left\langle\sigma_{x}\right\rangle_{\mu, h=h_{2}}-\left\langle\sigma_{x}\right\rangle_{\mu, h=h_{1}}\right] \leq \frac{4}{\beta\left|\Lambda_{L}\right|},
$$

where the dependence of the Gibbs expectation in the uniform field $h$ has been denoted explicitly. The infinite-volume limit of the variance of the overlap vanishes for almost all $h \in \mathbb{R}$ for any $\left(q_{i}\right)_{i=1, \cdots, N}$. Also in the exceptional case, the same way proves that the variance the overlap defined by (12) vanishes. 
Lemma 3.6 The following variance vanishes in the infinite-volume limit

$$
\lim _{L \rightarrow \infty} \mathbb{E}\left\langle\delta \xi_{L}^{2}\right\rangle_{\mu}=0
$$

for almost all $\mu \in \mathbb{R}$, where $\delta \xi_{L}:=\xi_{L}-\left\langle\xi_{L}\right\rangle_{\mu}$.

Proof. The variance $\mathbb{E}\left\langle\delta \xi_{L}^{2}\right\rangle_{\mu}$ is represented in the derivative of the one point function

$$
\begin{aligned}
& \mathbb{E}\left\langle\delta \xi_{L}^{2}\right\rangle_{\mu}=\frac{1}{\left|\Lambda_{L}\right|^{2}} \sum_{x, y \in \Lambda} \mathbb{E} g_{x} g_{y}\left(\left\langle\sigma_{x} \sigma_{y}\right\rangle_{\mu}-\left\langle\sigma_{x}\right\rangle_{\mu}\left\langle\sigma_{y}\right\rangle_{\mu}\right) \\
& =\frac{1}{\left|\Lambda_{L}\right|^{2} \beta} \frac{\partial}{\partial \mu} \sum_{x \in \Lambda_{L}} \mathbb{E} g_{x}\left\langle\sigma_{x}\right\rangle_{\mu}
\end{aligned}
$$

Integration over $\mu \in\left(\mu_{1}, \mu_{2}\right)$ yields

$$
\begin{aligned}
& \int_{\mu_{1}}^{\mu_{2}} d \mu \mathbb{E}\left\langle\delta \xi_{L}^{2}\right\rangle_{\mu}=\frac{1}{\left|\Lambda_{L}\right|^{2} \beta} \sum_{x \in \Lambda_{L}} \mathbb{E} g_{x}\left(\left\langle\sigma_{x}\right\rangle_{\mu_{2}}-\left\langle\sigma_{x}\right\rangle_{\mu_{1}}\right) \\
& =\frac{\beta}{\left|\Lambda_{L}\right|^{2} \beta} \sum_{x \in \Lambda_{L}} \mathbb{E}\left(\mu_{2}-\mu_{2}\left\langle\sigma_{x}\right\rangle_{\mu_{2}}^{2}-\mu_{1}+\mu_{1}\left\langle\sigma_{x}\right\rangle_{\mu_{1}}^{2}\right) \leq \frac{\beta\left(\left|\mu_{2}\right|+\left|\mu_{1}\right|\right)}{\left|\Lambda_{L}\right| \beta} .
\end{aligned}
$$

The limit vanishes

$$
\lim _{L \rightarrow \infty} \int_{\mu_{1}}^{\mu_{2}} d \mu \mathbb{E}\left\langle\delta \xi_{L}^{2}\right\rangle_{\mu}=0 .
$$

Since the integration interval is arbitrary and the integrand has a uniform bound, this completes the proof. Also in the exceptional case defined by (18), the same way proves that the variance of $\xi_{L}$ in the infinite-volume limit.

To show a bound on the following deviation of $\xi_{L}$, Lemma 3.4 and Lemma 3.6 and.

Lemma 3.7 The following limit vanishes

$$
\lim _{L \rightarrow \infty} \mathbb{E}\left\langle\left|\Delta \xi_{L}\right|\right\rangle_{\mu}=0
$$

for almost all $\mu \in \mathbb{R}$, where $\Delta \xi_{L}:=\xi_{L}-\mathbb{E}\left\langle\xi_{L}\right\rangle_{\mu}$

Proof.

$$
\begin{aligned}
& \mathbb{E}\left\langle\left|\Delta \xi_{L}\right|\right\rangle_{\mu}=\mathbb{E}\left\langle\left|\xi_{L}-\left\langle\xi_{L}\right\rangle_{\mu}+\left\langle\xi_{L}\right\rangle_{\mu}-\mathbb{E}\left\langle\xi_{L}\right\rangle\right|\right\rangle_{\mu} \\
& \leq \mathbb{E}\left\langle\left|\delta \xi_{L}\right|\right\rangle_{\mu}+\mathbb{E}\left|\left\langle\Delta \xi_{L}\right\rangle_{\mu}\right| \leq \sqrt{\mathbb{E}\left\langle\delta \xi_{L}^{2}\right\rangle_{\mu}}+\mathbb{E}\left|\left\langle\Delta \xi_{L}\right\rangle_{\mu}\right|
\end{aligned}
$$

Lemma 3.4 and Lemma 3.6 imply

$$
\lim _{L \rightarrow \infty} \int_{\mu_{1}}^{\mu_{2}} d \mu \mathbb{E}\left\langle\left|\Delta \xi_{L}\right|\right\rangle_{\mu}=0
$$

The integrand in the left hand side vanishes in the infinite-volume limit for almost all $\mu \in \mathbb{R}$. This completes the proof.

The Ghirlanda-Guerra identities are well-known as useful identities for disordered spin systems [5, 4, 7, 8, 9, 12, 15, 27]. The following lemma gives the Ghirlanda-Guerra identities for an arbitrary bounded function of spin configurations for almost all $\mu \in \mathbb{R}$.

Lemma 3.8 Let $f: \Sigma_{L}^{n} \rightarrow \mathbb{R}$ be a bounded measurable function of $n$ replicated spin configurations. For almost all $\mu \in \mathbb{R} \backslash\{0\}$, the following identity is valid in the infinite-volume limit of the perturbed model

$$
\lim _{L \rightarrow \infty}\left[\sum_{a=2}^{n} \mathbb{E}\left\langle R_{1, a} f\right\rangle_{\mu}-n \mathbb{E}\left\langle R_{1, n+1} f\right\rangle_{\mu}+\mathbb{E}\left\langle R_{1,2}\right\rangle_{\mu} \mathbb{E}\langle f\rangle_{\mu}\right]=0 .
$$


Proof. The following expectation has a bound

$$
\left|\mathbb{E}\left\langle\Delta \xi_{L} f\right\rangle_{\mu}\right| \leq \mathbb{E}\left\langle\left|\Delta \xi_{L}\right||f|\right\rangle_{\mu} \leq \sup _{\sigma \in \Sigma_{L}^{n}}|f(\sigma)| \mathbb{E}\left\langle\left|\Delta \xi_{L}\right|\right\rangle_{\mu}
$$

Since $f$ is bounded, the limit vanishes

$$
\lim _{L \rightarrow \infty}\left|\mathbb{E}\left\langle\Delta \xi_{L} f\right\rangle_{\mu}\right|=0 .
$$

The calculation of the left hand side gives

$$
\mathbb{E}\left\langle\Delta \xi_{L} f\right\rangle_{\mu}=\beta \mu\left[\sum_{a=2}^{n} \mathbb{E}\left\langle R_{1, a} f\right\rangle_{\mu}-n \mathbb{E}\left\langle R_{1, n+1} f\right\rangle_{\mu}+\mathbb{E}\left\langle R_{1,2}\right\rangle_{\mu} \mathbb{E}\langle f\rangle_{\mu}\right] .
$$

Since the left-hand side vanishes in the infinite-volume limit, the right-hand side also vanishes for almost all $\mu \in \mathbb{R} \backslash\{0\}$. This completes the proof.

Next, we prove the continuity of $\mathbb{E}\left\langle R_{1,2}\right\rangle_{\mu}$ and $\mathbb{E}\left\langle R_{1,2}\right\rangle_{\mu}^{2}$ at $\mu=0$.

Lemma 3.9 The following limit is identical to that at $\mu=0$

$$
\lim _{\mu \rightarrow 0} \lim _{L \rightarrow \infty} \mathbb{E}\left\langle R_{1,2}\right\rangle_{\mu}=\lim _{L \rightarrow \infty} \mathbb{E}\left\langle R_{1,2}\right\rangle_{0}
$$

for almost all $h \in \mathbb{R}$.

Proof. Represent the difference

$$
\begin{aligned}
& \left|\mathbb{E}\left\langle R_{1,2}\right\rangle_{\mu}-\mathbb{E}\left\langle R_{1,2}\right\rangle_{0}\right|=\left|\int_{0}^{\mu} d s \frac{\partial}{\partial s} \mathbb{E}\left\langle R_{1,2}\right\rangle_{s}\right| \\
& =\frac{2}{\left|\Lambda_{L}\right|}\left|\int_{0}^{\mu} d s \mathbb{E} \sum_{y \in \Lambda_{L}}\left\langle\sigma_{y}\right\rangle_{s} \frac{\partial}{\partial s}\left\langle\sigma_{y}\right\rangle_{s}\right| \\
& =\frac{2 \beta}{\left|\Lambda_{L}\right|}\left|\int_{0}^{\mu} d s \sum_{x, y \in \Lambda} \mathbb{E} g_{x}\left\langle\sigma_{y}\right\rangle_{s}\left(\left\langle\sigma_{x} \sigma_{y}\right\rangle_{s}-\left\langle\sigma_{x}\right\rangle_{s}\left\langle\sigma_{y}\right\rangle_{s}\right)\right| \\
& =\frac{2 \beta}{\left|\Lambda_{L}\right|}\left|\int_{0}^{\mu} d s \sum_{x, y \in \Lambda} \mathbb{E} \frac{\partial}{\partial g_{x}}\left\langle\sigma_{y}\right\rangle_{s}\left(\left\langle\sigma_{x} \sigma_{y}\right\rangle_{s}-\left\langle\sigma_{x}\right\rangle_{s}\left\langle\sigma_{y}\right\rangle_{s}\right)\right| \\
& =\frac{2 \beta^{2}}{\left|\Lambda_{L}\right|}\left|\int_{0}^{\mu} d s s \sum_{x, y \in \Lambda_{L}} \mathbb{E}\left(\left\langle\sigma_{x} \sigma_{y}\right\rangle_{s}-\left\langle\sigma_{x}\right\rangle_{s}\left\langle\sigma_{y}\right\rangle_{s}\right)\left(\left\langle\sigma_{x} \sigma_{y}\right\rangle_{s}-3\left\langle\sigma_{x}\right\rangle_{s}\left\langle\sigma_{y}\right\rangle_{s}\right)\right| \\
& \leq \frac{2 \beta^{2}}{\left|\Lambda_{L}\right|} \int_{0}^{\mu} d s s \sum_{x, y \in \Lambda_{L}} \mathbb{E}\left|\left\langle\sigma_{x} \sigma_{y}\right\rangle_{s}-\left\langle\sigma_{x}\right\rangle_{s}\left\langle\sigma_{y}\right\rangle_{s}\right|\left|\left\langle\sigma_{x} \sigma_{y}\right\rangle_{s}-3\left\langle\sigma_{x}\right\rangle_{s}\left\langle\sigma_{y}\right\rangle_{s}\right| \\
& \leq \frac{8 \beta^{2}}{\left|\Lambda_{L}\right|} \int_{0}^{\mu} d s s \sum_{x, y \in \Lambda_{L}} \mathbb{E}\left\langle\sigma_{x} ; \sigma_{y}\right\rangle_{s}=\frac{8 \beta}{\left|\Lambda_{L}\right|} \int_{0}^{\mu} d s s \mathbb{E} \sum_{x \in \Lambda_{L}} \frac{\partial}{\partial h}\left\langle\sigma_{x}\right\rangle_{s}
\end{aligned}
$$

The FKG inequality has been used. Therefore, the integral over $h$ in an arbitrary interval becomes

$$
\begin{aligned}
& \int_{h_{1}}^{h_{2}} d h\left|\mathbb{E}\left\langle R_{1,2}\right\rangle_{\mu, h}-\mathbb{E}\left\langle R_{1,2}\right\rangle_{0, h}\right| \leq \frac{8 \beta}{\left|\Lambda_{L}\right|} \int_{0}^{\mu} d s s \mathbb{E} \sum_{x \in \Lambda}\left[\left\langle\sigma_{x}\right\rangle_{s, h=h_{2}}-\left\langle\sigma_{x}\right\rangle_{s, h=h_{1}}\right] \\
& \leq \frac{8 \beta}{\left|\Lambda_{L}\right|} \int_{0}^{\mu} d s s \mathbb{E} \sum_{x \in \Lambda} 2 \leq 8 \beta \mu^{2}
\end{aligned}
$$

where we have represented the dependence of the Gibbs expectation in the uniform field $h$ explicitly. Therefore, the integrand vanishes in the infinite-volume limit. Also in the exceptional case defined by (12), this is proven in the same way. 
Lemma 3.10 The following limit is identical to that at $\mu=0$

$$
\lim _{\mu \rightarrow 0} \lim _{L \rightarrow \infty} \mathbb{E}\left\langle R_{1,2}\right\rangle_{\mu}^{2}=\lim _{L \rightarrow \infty} \mathbb{E}\left\langle R_{1,2}\right\rangle_{0}^{2}
$$

for almost all $h \in \mathbb{R}$.

Proof. As in the proof of Lemma 3.9 .

$$
\begin{aligned}
& \left|\mathbb{E}\left\langle R_{1,2}\right\rangle_{\mu}^{2}-\mathbb{E}\left\langle R_{1,2}\right\rangle_{0}^{2}\right|=\left|\int_{0}^{\mu} d s \frac{\partial}{\partial s} \mathbb{E}\left\langle R_{1,2}\right\rangle_{s}^{2}\right| \\
& =2\left|\int_{0}^{\mu} d s \mathbb{E}\left\langle R_{1,2}\right\rangle_{s} \frac{\partial}{\partial s}\left\langle R_{1,2}\right\rangle_{s}\right|=\left.2\left|\int_{0}^{\mu} d s \mathbb{E}\left\langle R_{1,2}\right\rangle_{s} \frac{\partial}{\partial s}\right| \Lambda_{L}\right|^{-1} \sum_{y \in \Lambda_{L}}\left\langle\sigma_{y}\right\rangle_{s} \mid \\
& =4\left|\Lambda_{L}\right|^{-1}\left|\int_{0}^{\mu} d s \mathbb{E}\left\langle R_{1,2}\right\rangle_{s} \sum_{y \in \Lambda_{L}}\left\langle\sigma_{y}\right\rangle_{s} \frac{\partial}{\partial s}\left\langle\sigma_{y}\right\rangle_{s}\right| \\
& =4 \beta\left|\Lambda_{L}\right|^{-1}\left|\int_{0}^{\mu} d s \mathbb{E} \sum_{x, y \in \Lambda} g_{x}\left\langle R_{1,2}\right\rangle_{s}\left\langle\sigma_{y}\right\rangle_{s}\left\langle\sigma_{x} ; \sigma_{y}\right\rangle_{s}\right| \\
& =4 \beta\left|\Lambda_{L}\right|^{-1}\left|\int_{0}^{\mu} d s \mathbb{E} \sum_{x, y \in \Lambda} \frac{\partial}{\partial g_{x}}\left\langle R_{1,2}\right\rangle_{s}\left\langle\sigma_{y}\right\rangle_{s}\left\langle\sigma_{x} ; \sigma_{y}\right\rangle_{s}\right| \\
& =4 \beta\left|\Lambda_{L}\right|^{-2}\left|\int_{0}^{\mu} d s \mathbb{E} \sum_{x, y, z \in \Lambda}\left(\left\langle\sigma_{z}\right\rangle_{s}^{2} \frac{\partial}{\partial g_{x}}\left\langle\sigma_{y}\right\rangle_{s}\left\langle\sigma_{x} ; \sigma_{y}\right\rangle_{s}+\left\langle\sigma_{y}\right\rangle_{s}\left\langle\sigma_{x} ; \sigma_{y}\right\rangle_{s} \frac{\partial}{\partial g_{x}}\left\langle\sigma_{z}\right\rangle_{s}^{2}\right)\right| \\
& =4 \beta^{2}\left|\Lambda_{L}\right|^{-2} \mid \int_{0}^{\mu} d s s \mathbb{E} \sum_{x, y, z \in \Lambda}\left(\left\langle\sigma_{z}\right\rangle_{s}^{2}\left\langle\sigma_{x} ; \sigma_{y}\right\rangle_{s}\left(\left\langle\sigma_{x} \sigma_{y}\right\rangle_{s}-3\left\langle\sigma_{x}\right\rangle_{s}\left\langle\sigma_{y}\right\rangle_{s}\right)\right. \\
& \left.+2\left\langle\sigma_{z}\right\rangle_{s}\left\langle\sigma_{y}\right\rangle_{s}\left\langle\sigma_{x} ; \sigma_{y}\right\rangle_{s}\left\langle\sigma_{x} ; \sigma_{z}\right\rangle_{s}\right) \mid \\
& \leq 4 \beta^{2}\left|\Lambda_{L}\right|^{-2} \int_{0}^{\mu} d s s \mathbb{E} \sum_{x, y, z \in \Lambda}\left(\left\langle\sigma_{z}\right\rangle_{s}^{2}\left|\left\langle\sigma_{x} ; \sigma_{y}\right\rangle_{s}\right|\left|\left\langle\sigma_{x} \sigma_{y}\right\rangle_{s}-3\left\langle\sigma_{x}\right\rangle_{s}\left\langle\sigma_{y}\right\rangle_{s}\right|\right) \\
& \left.+2\left|\left\langle\sigma_{z}\right\rangle_{s}\right|\left|\left\langle\sigma_{y}\right\rangle_{s}\right|\left|\left\langle\sigma_{x} ; \sigma_{y}\right\rangle_{s}\right|\left|\left\langle\sigma_{x} ; \sigma_{z}\right\rangle_{s}\right|\right) \\
& \leq 4 \beta^{2}\left|\Lambda_{L}\right|^{-2} \int_{0}^{\mu} d s s \mathbb{E} \sum_{x, y, z \in \Lambda}\left(\left|\left\langle\sigma_{x} ; \sigma_{y}\right\rangle_{s}\right|\left|\left\langle\sigma_{x} \sigma_{y}\right\rangle_{s}-3\left\langle\sigma_{x}\right\rangle_{s}\left\langle\sigma_{y}\right\rangle_{s}\right|\right. \\
& \left.+2\left|\left\langle\sigma_{x} ; \sigma_{y}\right\rangle_{s}\right|\left|\left\langle\sigma_{x} ; \sigma_{z}\right\rangle_{s}\right|\right) \leq 4 \beta^{2}\left|\Lambda_{L}\right|^{-2} \int_{0}^{\mu} d s s \mathbb{E} \sum_{x, y, z \in \Lambda}\left(4\left\langle\sigma_{x} ; \sigma_{y}\right\rangle_{s}+4\left\langle\sigma_{x} ; \sigma_{y}\right\rangle_{s}\right) \\
& =32 \beta^{2}\left|\Lambda_{L}\right|^{-1} \int_{0}^{\mu} d s s \mathbb{E} \sum_{x, y \in \Lambda_{L}}\left\langle\sigma_{x} ; \sigma_{y}\right\rangle_{s}=32 \beta\left|\Lambda_{L}\right|^{-1} \int_{0}^{\mu} d s s \mathbb{E} \sum_{x \in \Lambda_{L}} \frac{\partial}{\partial h}\left\langle\sigma_{x}\right\rangle_{s} \\
& \\
& =
\end{aligned}
$$

The FKG inequality has been used. Therefore, the integral over $h$ in an arbitrary interval becomes

$$
\begin{aligned}
& \int_{h_{1}}^{h_{2}} d h\left|\mathbb{E}\left\langle R_{1,2}\right\rangle_{\mu, h}^{2}-\mathbb{E}\left\langle R_{1,2}\right\rangle_{0, h}^{2}\right| \\
& \leq 32 \beta\left|\Lambda_{L}\right|^{-1} \int_{0}^{\mu} d s s \mathbb{E} \sum_{x \in \Lambda_{L}}\left[\left\langle\sigma_{x}\right\rangle_{\mu, h=h_{2}}-\left\langle\sigma_{x}\right\rangle_{\mu, h=h_{1}}\right] \\
& \leq 32 \beta\left|\Lambda_{L}\right|^{-1} \int_{0}^{\mu} d s s \mathbb{E} \sum_{x \in \Lambda_{L}} 2 \leq 32 \beta \mu^{2},
\end{aligned}
$$

where we have represented the dependence of the Gibbs expectation in the uniform field $h$ explicitly. Also in the exceptional case defined by (12), this is proven in the same way. $\square$ 


\subsection{Proof of Theorem 2.1}

First, let us remark the relation between three variances of the overlap. As discussed by Chatterjee [4], the Ghirlanda-Guerra identities (84) for $n=2, f=R_{1,2}$ and $n=3, f=R_{2,3}$ give the following relations for almost all $\mu \in \mathbb{R}$.

$$
\begin{aligned}
& 3 \lim _{L \rightarrow \infty}\left[\mathbb{E}\left\langle R_{1,2}{ }^{2}\right\rangle_{\mu}-\mathbb{E}\left\langle R_{1,2}\right\rangle_{\mu}^{2}\right] \\
= & 2 \lim _{L \rightarrow \infty}\left[\mathbb{E}\left\langle R_{1,2}{ }^{2}\right\rangle_{\mu}-\left(\mathbb{E}\left\langle R_{1,2}\right\rangle_{\mu}\right)^{2}\right] \\
= & 6 \lim _{L \rightarrow \infty}\left[\mathbb{E}\left\langle R_{1,2}\right\rangle_{\mu}^{2}-\left(\mathbb{E}\left\langle R_{1,2}\right\rangle_{\mu}\right)^{2}\right] .
\end{aligned}
$$

Since the first line (112) vanishes from Lemma 3.5, all lines vanishes for almost all $\mu \in \mathbb{R}$. Therefore Lemma 3.9 and Lemma 3.10 imply

$$
\lim _{L \rightarrow \infty} \mathbb{E}\left\langle R_{1,2}\right\rangle_{0}^{2}=\lim _{\mu \rightarrow 0} \lim _{L \rightarrow \infty} \mathbb{E}\left\langle R_{1,2}\right\rangle_{\mu}^{2}=\lim _{\mu \rightarrow 0} \lim _{L \rightarrow \infty}\left(\mathbb{E}\left\langle R_{1,2}\right\rangle_{\mu}\right)^{2}=\lim _{L \rightarrow \infty}\left(\mathbb{E}\left\langle R_{1,2}\right\rangle_{0}\right)^{2}
$$

for almost all $h \in \mathbb{R}$. Since Lemma 3.5 implies

$$
\lim _{L \rightarrow \infty}\left(\mathbb{E}\left\langle R_{1,2}^{2}\right\rangle_{0}-\mathbb{E}\left\langle R_{1,2}\right\rangle_{0}^{2}\right)=0
$$

for almost all $h \in \mathbb{R}$, also the following variance of the overlap vanishes

$$
\lim _{L \rightarrow \infty}\left[\mathbb{E}\left\langle R_{1,2}{ }^{2}\right\rangle_{0}-\left(\mathbb{E}\left\langle R_{1,2}\right\rangle_{0}\right)^{2}\right]=0 .
$$

for almost all $h \in \mathbb{R}$. This completes the proof of Theorem 2.1.

\section{References}

[1] Aizenman, M., Contucci, P. : On the stability of quenched state in mean-field spin glass models. J. Stat. Phys. 92, 765-783(1997)

[2] Auffinger, A., Chen, W.:Universality of Chaos and Ultrametricity in Mixed $p$ Spin Models. Commun. Pure. Appl. Math. 69 2107-2130 (2016)

[3] Barbier, J., Chan, C. L., Macris, N.:Concentration of multi-overlaps for random ferromagnetic spin models. preprint, arXiv:1901.06521(2019)

[4] Chatterjee, S.: Absence of replica symmetry breaking in the random field Ising model. Commun. Math .Phys. 337, 93-102(2015)

[5] Chatterjee, S.: The Ghirlanda-Guerra identities without averaging. preprint, arXiv:0911.4520 (2009).

[6] Chen, Y.-T.:Universality of Ghirlanda-Guerra identities and spin distributions in mixed p-spin models. Ann. Inst. H. Poincarè Probab. Statis. 55, 528-550, (2019)

[7] Contucci, P., Giardinà, C. : Spin-glass stochastic stability: A rigorous proof. Ann. Inst. H. Poincarè, Probab. Statis. 6, 915-923, (2005)

[8] Contucci, P., Giardinà, C. : The Ghirlanda-Guerra identities. J. Stat. Phys. 126, 917$931,(2007)$

[9] Contucci, P., Giardinà, C. : Perspectives on spin glasses. Cambridge university press, 2012. 
[10] Contucci, P., Lebowitz, J. L. : Correlation inequalities for quantum spin systems with quenched centered disorder. J. Math. Phys. 51, 023302-1 -6 (2010)

[11] Fortuin,C. M., Kasteleyn P. W., Ginibre, J.: Correlation inequalities on some partially ordered sets.Commun. Math. Phys. 22, 89-103(1971).

[12] Ghirlanda, S., Guerra, F. : General properties of overlap probability distributions in disordered spin systems. Towards Parisi ultrametricity. J. Phys. A31, 9149-9155(1998)

[13] Griffiths, R. B. :Correlations in Ising Ferromagnets. I J. Math.Phys.8, 478 483 (1967)

[14] Griffiths, R. B. :Correlations in Ising Ferromagnets. II External Magnetic Fields. J. Math.Phys.8, 484 489 (1967)

[15] Itoi, C. :Universal nature of replica symmetry breaking in quantum systems with Gaussian disorder J. Stat. phys. 167 1262-1279 (2017)

[16] Itoi, C. :Absence of replica symmetry breaking in transverse and longitudinal random field Ising model J. Stat. phys. 170 684-699 (2018)

[17] Itoi, C. :Zero-variance of perturbation Hamiltonian density in perturbed spin systems. J. Stat. phys. 174 - (2019)

[18] Itoi, C. :Self-averaging of perturbation Hamiltonian density in perturbed spin systems. arXiv. 1908.09423

[19] Itoi, C and Utsunomiya, Y. :No replica symmetry breaking phase in the random field Ginzburg-Landau model, J. Math.Phys.60, 083304-1 083304- 19 (2019)

[20] Kelly, D. G., Sherman, S. :General Griffiths' Inequalities on Correlations in Ising Ferromagnets. J. Math. Phys. 9, 466 484 (1968)

[21] Krzakala, F., Ricci-Tersenghi, F. and Zdeborova, L. : Elusive Spin- Glass Phase in the Random Field Ising Model. Phys. Rev. Lett., 104, 207208 (2010).

[22] F. Krzakala, F. Ricci-Tersenghi, D. Sherrington and Zdeborova, L :No spin glass phase in ferromagnetic random field and random temperature scalar Ginzburg-Landau models J. Phys. A: Math. Theor. 44, 042003 (2011).

[23] Parisi, G. :A sequence of approximate solutions to the S-K model for spin glasses. J. Phys. A 13, L-115 (1980)

[24] Roldan, J. and Vira, R. :On the absence of replica symmetry breaking for the random field Ising model in the presence of a class of non-Gaussian disorders, arxXv:1811.07003v2 (2018)

[25] Sherrington, S., Kirkpatrick, S : Solvable model of spin glass. Phys. Rev. Lett. 35, 17921796, (1975).

[26] Talagrand, M. : The Parisi formula. Ann. Math. 163, 221-263 (2006).

[27] Talagrand, M. : Mean field models for spin glasses. Springer, Berlin (2011). 